\title{
Cost Effectiveness of Surgical Outreach Compared with Routine Surgeries at a Facility in Jos, Nigeria
}

\author{
Isichei M.W ${ }^{1}$, Misauno M.A ${ }^{1}$, Shitta A.H ${ }^{1}$, Isichei C.O ${ }^{2}$, Enwerem K.E \\ ${ }^{I}$ Department of surgery, University of Jos \\ ${ }^{2}$ Department of chemical Pathology, University of Jos \\ ${ }^{3}$ Faith Alive Foundation hospital
}

\begin{abstract}
This is a cost effectiveness analysis study of patients whose surgical conditions were treated during a free surgical outreach program at a facility Jos, Plateau State, Nigeria over a four week period in, 2013.A total of 264 surgical operations were carried out on 253 patients, Males (56\%) and females (44\%). The mean age of beneficiaries was 38years $\pm 17.2 S D$, the youngest being 10months and the oldest being 70years. Surgeries done were herniorrhaphies and hydrocelectomies 79(29.92\%), appendicectomy 28(10.52\%), Thyroidectomies 7(2.65\%) Laparotomies 5(1.89\%), Obstetric and Gynaecoloical surgeries 33(12.50\%), Pediatric 15(5.78) and lump excisions 97(36.84\%). Two-thirds (66.67\%) of cases were done under local anesthesia, spinal and general anesthesia were used in $8.77 \%$ and $24.56 \%$ of cases respectively. No mortality was recorded, surgical site infection 7 (2.65\%), YB;N routine procedure N5, 754,732.72(\$35,967.08), a unit price of 21,798.23(\$136.24). Surgical outreach is a cost effective method of surgery compared to routine referral system and it also reduces surgical burden in a resource poor communities.
\end{abstract}

Keywords: cost, effectiveness, outreach, referral, Surgical

\section{Introduction}

Surgical conditions account for a significant portion of a population's burden of disease. The World Health Organization estimates that surgical conditions contribute to $11 \%$ of world's total Disability Life Adjusted Years (DALYs)(1). Sub Saharan Africa is undergoing a double burden disease pattern, the burden of communicable and no communicable diseases (2). In this era of epidemiological disease transition, much of the non-communicable disease burden accounts for more than double the combined mortality from infectious disease, maternal and perinatal conditions and malnutrition (3). Some of these diseases are amenable to surgical treatment.

The presence of a huge backlog of untreated surgical diseases in the scenario where conservative treatment is not readily available and/or accessible sets the pace for a high burden of surgical disease in low middle income settings. [Muray and Lopez 1996] A fairly recent estimate shows that only $3.5 \%$ of the total surgical procedures done annually occur in the developing world - a statistic suggesting a large unaddressed disease burden (4) [Weiser et al, 2008]

Surgical outreach is designed to help those who are unable to get there surgeries done because they are less privileged. The outreach cost of surgery maybe fully or partially sponsored by the organizing body. This include but not limited to cost of space, transportation, equipment, laboratory tests, consumables, drugs, feeding and stipend for volunteers. Key informant interview of patients presenting to the outreach program reviled lack of financial resources to pay for surgery at normal clinic procedure was the major reason for not having their surgeries done at the time of diagnosis. The facility is a non-governmental, non-profit, and medical and social services organization which offers free surgical services to clients although clients have to procure the surgical consumables required for the surgery. During surgical outreaches however the facility bares all costs.

We analyzed the total cost and the unit cost of free outreach surgeries done at the facility compared with unit cost of routine surgical procedures with a view to assess the cost effectiveness of surgical outreach in our facility.

\section{Methods}

This is a prospective study conducted at a health facility in Jos North Local Government Area of Plateau State. Patients were examined, evaluated, prepared for and had surgery at the facility. Other investigations such as ultrasonography, radiographs, and assays were done on selected patients. Day surgeries were seen on post operative day five while those requiring admission were kept till fit for discharge. Informed consent was obtained from patient in the presence of a relative who co-signed as a witness. Where required, blood was pre-screened and cross-matched from consented donors (usually patients' relatives). The cost of items purchased, investigations, consultations, surgical items used, pre and post operative care, feeding of volunteers 
and stipend were recorded and analyzed by the account person in the facility. This was compared with cost of purchase of surgical items (facilities standard list of items) by patients for minor, medium and major surgeries in addition to cost incurred by the facility per patient for equal number of patients and type of surgeries.

\section{Results}

A total of 264 surgical operations were carried out on 253 patients. There were slightly more males (56\%) than females $(44 \%)$. The mean age of beneficiaries was 38 years $\pm 17.2 \mathrm{SD}$, the youngest being $10 \mathrm{months}$ and the oldest being 70years. Surgeries done were herniorrhaphies/hydrocelectomies 79(29.92\%), appendicectomy 28(10.52\%), Thyroidectomies 7(2.65\%) Laparotomies 5(1.89\%), Obstetric and Gynaecoloical surgeries 33(12.50\%), Pediatric 15(5.78) and lump excisions $97(36.84 \%)$ dominated the surgical procedures done. Two-thirds $(66.67 \%)$ of cases were done under local anesthesia, spinal and general anesthesia were used in $8.77 \%$ and $24.56 \%$ of cases respectively. No mortality recorded, surgical site infection 7 $(2.65 \%)$, scrotal hematoma $2(0.04 \%)$. Total outreach cost N2, 616700(\$16,354.375), a unit price of N9911.74 (\$61.95). Cost of routine procedure for equal number of patients over 13months N5, 754,732.72(\$35,967.08), a unit price of 21,798.23(\$136.24)

\section{Discussion}

The main finding in this study is the considerable difference between the unit cost of surgery during routine procedures compared to the unit cost of surgery during outreaches. The unit cost at outreaches is just about half amount spent on routine surgeries. The pooling together of resources and personnel has a good economy of scale effect that contributed to lowering of the unit cost. Kifle et al found in similar studies that outreach cost of specialist care was 1.45times more cost effective compared to referral system(5-6)

The outreach had a multifaceted outcome; it effectively utilized human resource in a way that made possible close interaction with the community which would not have been possible in routine referral system, and utilized less material resources including time to achieve more than usually obtainable result compared with routine referral system. The outreach was conduct over a four weeks period; number of persons and operations was equal number of cases that took 13 months of routine surgery to achieve. Turner et al did a similar study conducting eye surgery outreach in Australia, found an increased surgical case rate compared to routine eye surgeries (7-8).Such surgical outreaches also contribute to the reduction in disease burden of target populations as individuals who benefit from such interventions would otherwise have remained with their diseases. Ojo et al found that there is reduction in disease burden to the community in rural areas following surgical outreaches (9$10)$.

The morbidity and mortality is very low, despite the high turnover. This could have occurred as a result of coming together of complementary skills working as a team leading to a better outcome. Klein et al found in their study all operations were successfully performed, with very low adverse outcomes (morbidity, mortality) and good quality of life for all the subjects. They found that safety and cost-effectiveness of cardiothoracic surgery under primitive conditions were demonstrated (11-12)

\section{Conclusion}

Surgical outreach is a cost effective method of surgery compared to routine referral system and it also reduces surgical burden in a resource poor communities.

\section{References}

[1]. Weiser TG, Regenbogen SE, Thompson KD, et al. An estimation of the global volume of surgery: a modeling strategy based on available data. Lancet 2008; 372: 139-144

[2]. Maher D, Smeeth L, Sekajugo J. Health transition in Africa: practical policy proposals for primary care. Bull World Health Organ. 2010 Dec 1;88(12):943-8.

[3]. Ozgediz D, Jamison D, Cherian M, McQueen K. The burden of surgical conditions and access to surgical care in low and middle income countries. Bulletin of the World Health Organisation 2008; 86(8): 646-647.

[4]. Daar AS, Singer PA, Persad DL, Pramming SK, Matthews DR, Beaglehole R, et al. Grand challenges in chronic non-communicable diseases.Nature. 2007 Nov 22;450(7169). PubMed PMID: WOS:000251158500032.

[5]. Kifle YA, Nigatu TH. Cost-effectiveness analysis of clinical specialist outreach as compared to referral system in Ethiopia: an economic evaluation. Cost Eff Resour Alloc. 2010;8(1):13.

[6]. Casey KM. The global impact of surgical volunteerism. Surg Clin North Am. 2007 Aug;87(4):949-60, ix.-6

[7]. Turner AW, Mulholland WJ, Taylor HR. Coordination of outreach eye services in remote Australia. Clin Experiment Ophthalmol. 2011 May-Jun;39(4):344-9.

[8]. Castle PE, Rausa A, Walls T, Gravitt PE, Partridge EE, Olivo V, et al. Comparative community outreach to increase cervical cancer screening in the Mississippi Delta. Prev Med. 2011 Jun;52(6):452-5.

[9]. Ojo E, Okoi E, Umoiyoho AJ, Nnamonu M. Surgical outreach program in poor rural Nigerian communities. Rural Remote Health. 2013 Jan-Mar;13(1):2200.

[10]. 10.Chang HC, Hsiung HY, Chen SI, Yen AM, Chen TH. Comparison of a community outreach service with opportunity screening for cervical cancer using Pap smears. J Public Health (Oxf). 2007 Jun;29(2):165-72.

[11]. Klein M, Ramoroko SP, Jacobs AG, Bomela MD, Mokhobo KP, Mohlala ML. The Cardiothoracic Outreach Programme--a pilot project. S Afr Med J. 1996 Dec;86(12):1533-5.9

[12]. Bonner J. Clinical research outreach: the practitioner's contribution. J Small Anim Pract. 2006 Aug;47(8):490-1. 